\title{
Neonatal Kawasaki Disease: A Case Report
}

\author{
Mohamed A. Huneif ${ }^{*}$, Ayed A. Shati², Abdu M. Abudiah ${ }^{3}$, Abdullah M. Aljaser ${ }^{3}$, \\ Mansour Y. Otaif ${ }^{4}$ \\ ${ }^{1}$ Department of Child Health, College of Medicine, Najran University, Najran, KSA \\ ${ }^{2}$ Department of Child Health, College of Medicine, King Khalid University, Abha, KSA \\ ${ }^{3}$ Department of Pediatric Rheumatology, King Faisal Specialist Hospital, Ministry of Health, Riyadh, KSA \\ ${ }^{4}$ Department of Pediatric Neurology, King Faisal Specialist Hospital, Ministry of Health, Riyadh, KSA \\ Email: ${ }^{*}$ Huneif@hotmail.com
}

Received 10 February 2015; accepted 21 April 2015; published 24 April 2015

Copyright (C) 2015 by authors and Scientific Research Publishing Inc.

This work is licensed under the Creative Commons Attribution International License (CC BY).

http://creativecommons.org/licenses/by/4.0/

(c) (i) Open Access

\section{Abstract}

Objective: To report a case of Kawasaki disease (KD) with unusual age at presentation. Case Report: A 26-day-old Saudi boy presented with fever, irritability and poor feeding for $\mathbf{3}$ days. There was right cervical lymph node enlargement. There was mild throat congestion and bilateral congested tympanic membrane. The liver was palpable. Otherwise, there were no more abnormal physical findings. Laboratory findings revealed high erythrocyte sedimentation rate and positive $\mathrm{C}$-reactive protein. The patient was admitted into an isolation room and antibiotics were started. On the $5^{\text {th }}$ day, the condition of the patient deteriorated. Ampicillin was discontinued and vancomycin was started till getting the results of the culture sensitivity. On the $7^{\text {th }}$ day, he developed diaper rash and fever spikes continued. On the $8^{\text {th }}$ day, the patient developed swelling, redness and hotness of the right hand. Possibility of sepsis was considered and the administered antibiotics were imipenem and vancomycin, while cefotaxime was discontinued. On the $12^{\text {th }}$ day, the patient developed anemia and leukocytosis. Gamma glutamyl transpeptidase was high. On the $15^{\text {th }}$ day, the patient started to develop maculopapular rash on the trunk and desquamation of both hands and feet with swelling, cracked lips, bilateral conjunctival injection and unilateral neck lymph node swelling. So, the infant's condition was clinically diagnosed as KD. Aspirin was started and 2 doses of intravenous immunoglobulins were given. On the $18^{\text {th }}$ day, the condition of patient improved and the fever subsided. Conclusions: The diagnosis of KD among neonates is a clinical challenge. Diagnosis is based on clinical criteria after the exclusion of other diseases presenting with high persistent fever. Early treatment by administering intravenous immunoglobulins and aspirin can help prevent cardiac complications. There is a pressing need to raise awareness among pediatricians about this disease.

\section{Keywords}

Kawasaki Disease, Neonate, Skin Desquamation, Strawberry Tongue, Immunoglobulin,

\footnotetext{
${ }^{*}$ Corresponding author.
} 


\section{Cardiovascular Complications}

\section{Introduction}

Kawasaki Disease (KD) is an acute febrile illness, which often affects children younger than five years. It involves lymph nodes, skin, and mucous membranes inside the mouth, nose, and throat. Potentially, it can affect the heart and its larger arteries [1].

The exact etiology of KD remains unknown. However, evidence supports that it may be triggered by activation of immunocytes stimulated by toxins or superantigens, leading to increased levels of cytokines and activation of an arachidonic acid cascade. These inflammatory reactions result in diffuse vasculitis and subsequently development of acute clinical symptoms and cardiovascular complications [2].

$\mathrm{KD}$ is the commonest vasculitic illness of childhood and also the commonest cause of acquired heart disease in children in developed countries [3]. The disease has a world-wide distribution with a male preponderance, an ethnic bias toward Asian children (particularly East Asian), seasonality and occasional epidemics [4]. The incidence in Japan is 138/100,000 in children younger than 5 years, whereas in the USA, it is 17.1/100,000 and in the UK 8.1/100,000 [5].

The administration of a high dose of intravenous immunoglobulin and acetyl salicylic acid decreases the occurrence of coronary artery abnormalities and improves acute clinical manifestations [6].

$\mathrm{KD}$ is unusual in neonates. Of 105,755 patients with KD registered in Japan over 25 years, only six were neonates, with the youngest aged 20 days [7]. In this study, we report a case of KD with unusual age at presentation.

\section{Case Report}

A 26-day-old, Saudi boy from Abha City, Kingdom of Saudi Arabia, presented to the Emergency Department of Aseer Central Hospital with history of fever, irritability and poor feeding for 3 days prior to presentation. He was a full term baby, product of normal spontaneous vaginal delivery with uneventful pregnancy. He cried immediately after birth with weight of $3 \mathrm{~kg}$. Birth vaccination was given, and he was discharged home in a good condition. The baby remained well till 3 days prior to admission to our hospital, when he started to develop high grade fever $\left(39.5^{\circ} \mathrm{C}\right)$, which partially responded to antipyretics and cold sponges and was associated with poor feeding.

There was no history of contact with any sick or infected patients. There was no family history of similar illness. His family was living in Abha City with no history of travelling to an endemic area. The socioeconomic status of the family was high.

On physical examination, the baby was looking unwell, irritable, not in respiratory distress, nor dehydrated. He was not pale, jaundiced, or dysmorphic. He had high grade fever $\left(39.5^{\circ} \mathrm{C}\right)$. The heart rate was 165 beats/minute, the respiratory rate was 30 breaths/minute and the blood pressure was $87 / 42 \mathrm{mmHg}$. The oxygen saturation was $96 \%$ on room air. His growth parameters were on the $50^{\text {th }}$ percentile according to the Saudi growth charts (weight 4.2 $\mathrm{kg}$, length $53 \mathrm{~cm}$ and head circumference $37 \mathrm{~cm}$ ).

There were no skin rash or mouth ulcers. There was no ear or nasal discharge. There was right cervical lymph node enlargement $(1.5 \mathrm{~cm})$ with hotness and tenderness. There was mild throat congestion and bilateral congested tympanic membrane.

Eye examination was normal. Chest examination revealed good air entry bilaterally with no added sound. Cardiovascular system examination revealed normal first and second heart sounds with no murmur. Peripheral pulses were palpable. There was no abdominal distension or tenderness. The liver was palpable $3 \mathrm{~cm}$ below the right costal margin (liver span was $6 \mathrm{~cm}$ ). Otherwise, there were no more abnormal physical findings.

Central nervous system examination revealed that the anterior fontanel was open and flat, normal cranial nerves, power, tone and reflexes for upper and lower limbs with normal primitive reflexes. Skin and skeletal muscle system examinations were unremarkable.

Complete blood count was normal. Laboratory findings revealed high erythrocyte sedimentation rate of 42 $\mathrm{mm} /$ hour and positive C-reactive protein. Urea and electrolytes, blood gas, liver and renal function tests were 
normal. CSF results showed WBCs (8 cells), RBCs (Nil), proteins $(72 \mathrm{mg} / \mathrm{dL})$, glucose $(71 \mathrm{mg} / \mathrm{dL})$ and CSF culture was negative. Blood culture, urine analysis and culture and stool analysis and culture were all negative.

Chest X-ray was normal and abdominal ultrasonography revealed mild hepatomegaly with no focal liver lesion and mild hydronephrosis of the left kidney.

The patient was admitted into an isolation room and cefotaxime and ampicillin were started. Four days after admission, the patient showed slight improvement. There were no spikes of fever, feeding was good and the patient was active.

However, on the $5^{\text {th }}$ day, the patient developed 3 spikes of fever $\left(39^{\circ} \mathrm{C}\right)$, with 5 times' watery diarrhea and moderate dehydration and the condition of the patient deteriorated. Partial sepsis work-up was done. Antibiotics had been upgraded, ampicillin was discontinued and vancomycin was started till getting the results of the culture sensitivity.

On the $7^{\text {th }}$ day of admission, the patient developed diaper rash and fever spikes continued. On the $8^{\text {th }}$ day, the fever spikes continued and the patient developed swelling, redness and hotness of the right hand. Possibility of sepsis was considered and the administered antibiotics were imipenem and vancomycin, while cefotaxime was discontinued.

On the $12^{\text {th }}$ day of admission, the fever spikes were still occurring. The patient developed anemia (hemoglobin: $8 \mathrm{mg} / \mathrm{dL}$ ) and leukocytosis (WBC: $23 \times 10 / \mathrm{ml})$ and thrombocytosis $(900,000 \mathrm{mcL})$. The erythrocyte sedimentation rate became high $(90 \mathrm{~mm} / \mathrm{hr})$. Total and direct bilirubin were within normal values $(1.2 \mathrm{mg} / \mathrm{dl}$ and $0.7 \mathrm{mg} / \mathrm{dL}$, respectively), while gamma glutamyl transpeptidase was high (296 U/L). Peripheral blood smear revealed dimorphic anemia with hemolytic evidence along with absolute neutrophilia. Bone marrow examination revealed few cells showing hemophagocytosis with active bone marrow and no abnormal cells.

On the $15^{\text {th }}$ day after admission, the patient started to develop maculopapular rash on the trunk and desquamation of both hands and feet with swelling (Photo 1), cracked lips (Photo 2), bilateral conjunctival injection and unilateral neck lymph node swelling. Echocardiography showed normal structure and function with normal coronaries.

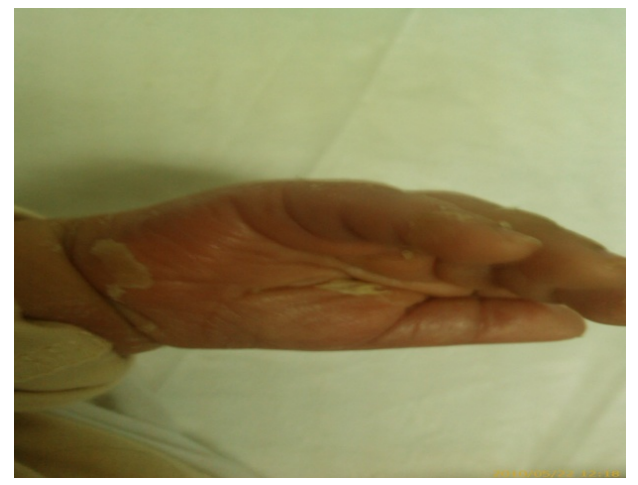

Photo 1. The hand of the patient showing desquamation.

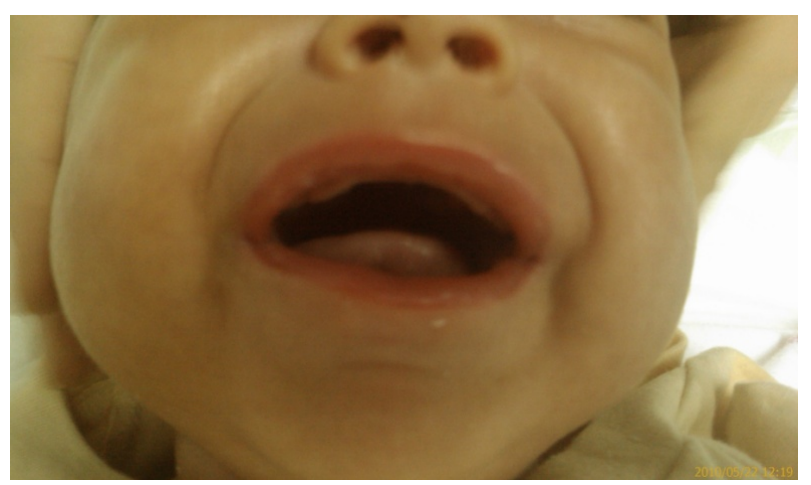

Photo 2. The mouth of patient showing cracked lips with strawberry tongue. 
So, the infant's condition was clinically diagnosed as KD. The diagnosis was based on the presence of fever persisting for more than 5 days in addition to: changes in extremities (i.e., acute erythema of palms, soles, edema of hands, feet, and peeling of fingers and toes); bilateral conjunctival injection without exudates; changes in lips and oral cavity (erythema of lips, cracking, strawberry tongue, diffuse injection of oral and pharyngeal mucosa); and cervical lymphadenopathy. The laboratory findings which supported the diagnosis of KD were: leucocytosis with neutrophilia, anemia, elevated erythrocyte sedimentation rate, elevated C-reactive protein and elevated serum gamma glutamyl transpeptidase.

Aspirin was started and two doses of intravenous immunoglobulins were given. On the $18^{\text {th }}$ day, the condition of patient improved and the fever subsided. The patient became active with good appetite, and then he was discharged after the second normal study of echocardiography.

The patient was seen in the Pediatric Cardiology Clinic after two weeks and the echocardiography showed normal study with regular follow up.

\section{Discussion}

This age at presentation of our 26-day case is quite rare, since KD mainly affects children aged 6 months to 4 years [8]. Chang et al. [9] added that KD is rarely reported in infants less than 3 months of age. The diagnosis in this age group is difficult because it is usually similar to other diseases.

Reaching a definite diagnosis for our case was a real challenge that took more than two weeks to be reached. Detailed history, physical examination, laboratory and radiological investigations were inconclusive. A definite diagnosis remained unsettled for two weeks.

This is in accordance with that noted by Burns and Glode [10], who reported that the diagnosis of KD is often delayed or may be missed entirely. There is no specific diagnostic test for KD. Its diagnosis relies on clinical features, some of which are common in children with fever due to other infections. Cimaz and Falcini [11] stated that the diagnosis is based on clinical criteria after the exclusion of other diseases presenting with high persistent fever.

Our case presented with high grade fever and irritability for 3 days. Despite there was not a single evidence for previous exposure to infection, our preliminary diagnosis was still "infection".

The role of exposure to infection in cases of KD has been considered. Nakamura et al. [12] stated that, although no specific pathogens for KD have been identified, its clinical and epidemiological features suggest that infectious agents might trigger the development of this disease. Microbiologically confirmed concurrent infections may be present in up to one-third of cases at presentation [13].

Moreover, the significant contribution of the innate immune system to the pathophysiology of the acute phase of the disease has been demonstrated in recent studies [14]. However, the etiology of KD is still unknown [15].

The patient was admitted into an isolation room. Management started with antipyretics and antibiotics (cefotaxime and ampicillin). The patient showed slight improvement after 4 days. However, on the $5^{\text {th }}$ day, the condition of the patient deteriorated. He developed spikes of fever that did not subside, with frequent watery diarrhea and moderate dehydration. Two days later, the patient developed diaper rash and fever spikes did not subside. Then, the patient developed swelling, redness and hotness of the right hand. The possibility of sepsis was considered. Imipenem and vancomycin were administered.

Zulian et al. [16] noted that some clinical features of KD are similar to other self-limiting febrile illnesses. Non-cardinal diagnostic features (e.g., diarrhea, vomiting, abdominal pain and arthralgia) are common and do not exclude the diagnosis.

Further deterioration occurred on the $12^{\text {th }}$ day of admission. In addition to the positive C-reactive protein, he developed anemia, leukocytosis and thrombocytosis, high ESR and Gamma glutamyltranspeptidase. On the $15^{\text {th }}$ day, the patient started to develop maculopapular rash on the trunk and desquamation of both hands and feet with swelling, cracked lips, bilateral conjunctival injection and unilateral neck lymph node swelling.

These findings are in according with that reported by Dillon et al. [17], who stated that KD is invariably associated with an inflammatory process, with elevation of ESR, CRP and white cell count.

Our patient's condition was clinically diagnosed as KD. This was based on the presence of fever persisting for more than 5 days in addition to: changes in extremities (i.e., acute erythema of palms, soles, edema of hands, feet, and peeling of fingers and toes); bilateral conjunctival injection without exudates; changes in lips and oral cavity (erythema of lips, cracking, strawberry tongue, diffuse injection of oral and pharyngeal mucosa); and cervical 
lymphadenopathy. The laboratory findings which supported the diagnosis of KD were leucocytosis with neutrophilia, anemia, elevated erythrocyte sedimentation rate, elevated C-reactive protein and elevated serum gamma glutamyltranspeptidase.

Coustasse et al. [18] noted that KD diagnostic criteria include high fever lasting 4 - 5 days, along with four or more of the following symptoms: rash, red eyes, red swollen and cracked lips, "strawberry" tongue, swollen hands and feet, swollen lymph nodes, and redness of the palms and soles of the feet.

Our case was managed by aspirin in addition to 2 doses of intravenous immunoglobulins. On the $18^{\text {th }}$ day, the condition of patient completely improved and the fever subsided. The patient became active with good appetite, and he was discharged after the second normal study of echocardiography, which showed normal structure and function with normal coronaries.

It is important to exclude any cardiac complication among cases of KD. Pannaraj et al. [8] stated that KD has surpassed acute rheumatic fever as the most common cause of pediatric acquired heart disease in the industrialized world, causing cardiac complications in up to $25 \%$ of untreated individuals. Gomard-Mennesson et al. [19] added that, with the potential for significant long-term cardiac sequelae, it is important that KD be clinically suspected, diagnosed and treated promptly. Several cases of myocardial infarction in young adults have been attributed to "missed" KD in childhood, which resulted in coronary artery aneurysms.

Burns [20] stated that KD should be treated in an inpatient pediatric facility. Intravenous immunoglobulin is the only proven therapy that improves coronary artery outcomes. It should be given at any time if the child is febrile or there are laboratory features of persisting inflammation. Intravenous immunoglobulin usually causes rapid defervescence and clinical improvement in the majority of cases. Gedalia [21] added that high-dose aspirin is an important adjunct to intravenous immunoglobulin therapy and is supposed to have an additive anti-inflammatory effect in KD.

\section{Conclusion}

Reaching the diagnosis in a case of neonatal KD is a clinical challenge at that age, since it rarely presents during the neonatal period. High fever is the main presenting symptom. Diagnosis is based on clinical criteria after the exclusion of other diseases presenting with high persistent fever. Treatment is mainly by administering intravenous immunoglobulins and aspirin, which proved to protect against development of cardiac complications. There is a pressing need to raise awareness among pediatricians about this disease so that affected children may get early appropriate treatment by prompt diagnosis and avoid cardiovascular complications.

\section{References}

[1] Newburger, J.W., Takahashi, M., Gerber, M.A., et al. Committee on Rheumatic Fever, Endocarditis, and Kawasaki disease, Council on Cardiovascular Disease in the Young, American Heart Association (2004) Diagnosis, Treatment, and Long-Term Management in Kawasaki Disease: A Statement for Health Professionals from the Committee on Rheumatic Fever, Endocarditis, and Kawasaki Disease, Council on Cardiovascular Disease in the Young, American Heart Association. Pediatrics, 114, 1708-1733. http://dx.doi.org/10.1542/peds.2004-2182

[2] Kim, H.J. and Lee, S.J. (2009) Two Cases of Kawasaki Disease Following Pneumonia. Korean Journal of Pediatrics, 52, 615-618. http://dx.doi.org/10.3345/kjp.2009.52.5.615

[3] Nakamura, Y., Yashiro, M., Uehara, R., et al. (2008) Epidemiologic Features of Kawasaki Disease in Japan: Results from the Nationwide Survey in 2005-2006. Journal of Epidemiology, 18, 167-172. http://dx.doi.org/10.2188/jea.JE2008001

[4] MacNeil, A., Holman, R., Yorita, K., et al. (2009) Evaluation of Seasonal Patterns of Kawasaki Syndrome- and Rotavirus-Associated Hospitalizations in California and New York, 2000-2005. BMC Pediatrics, 9, 65. http://dx.doi.org/10.1186/1471-2431-9-65

[5] Eleftheriou, D., Levin, M., Shingadia, D., et al. (2014) Management of Kawasaki Disease. Archives of Disease in Childhood, 99, 74-83. http://dx.doi.org/10.1136/archdischild-2012-302841

[6] Muzaffer, M.A. and Al-Mayouf, S.M. (2002) Pattern of Clinical Features of Kawasaki Disease. Saudi Medical Journal, 4, 409-412.

[7] Stanley, T.V. and Grimwood, K. (2002) Classical Kawasaki Disease in a Neonate. Archives of Disease in Childhood. Fetal and Neonatal Edition, 86, F135-F136. http://dx.doi.org/10.1136/fn.86.2.F135

[8] Pannaraj, P.S., Turner, C.L, Bastian, J.F. and Burns, J.C. (2004) Failure to Diagnose Kawasaki Disease at the Extremes of the Pediatric Age Range. Pediatric Infectious Disease Journal, 23, 789-791. 
http://dx.doi.org/10.1097/01.inf.0000134312.39744.a4

[9] Chang, F.Y., Hwang, B., Chen, S.J., Lee, P.C., Meng, C.C. and Lu, J.H. (2006) Characteristics of Kawasaki Disease in Infants Younger than Six Months of Age. Pediatric Infectious Disease Journal, 25, 241-244. http://dx.doi.org/10.1097/01.inf.0000202067.50975.90

[10] Burns, J.C. and Glode, M.P. (2004) Kawasaki Syndrome. Lancet, 364, 533-544. http://dx.doi.org/10.1097/01.inf.0000202067.50975.90

[11] Cimaz, R. and Falcini, F. (2003) An Update of Kawasaki Disease. Autoimmunity Review, 2, 258-263. http://dx.doi.org/10.1016/S1568-9972(03)00032-6

[12] Nakamura, Y., Yashiro, M., Uehara, R., et al. (2012) Epidemiologic Features of Kawasaki Disease in Japan: Results of the 2009-2010 Nationwide Survey. Journal of Epidemiology, 22, 216-221. http://dx.doi.org/10.2188/jea.JE20110126

[13] Benseler, S.M., McCrindle, B.W., Silverman, E.D., Tyrrell, P.N., Wong, J. and Yeung, R.S. (2005) Infections and Kawasaki Disease: Implications for Coronary Artery Outcome. Pediatrics, 116, e760-e766. http://dx.doi.org/10.1542/peds.2005-0559

[14] Ikeda, K., Yamaguchi, K., Tanaka, T., et al. (2010) Unique Activation Status of Peripheral Blood Monocuclear Cells at Acute Phase of Kawasaki Disease. Clinical \& Experimental Immunology, 160, 246-255. http://dx.doi.org/10.1111/j.1365-2249.2009.04073.x

[15] Leonardi, S., Miraglia del Giudice, M., La Rosa, M. and Bellanti, J. (2007) Atopic Disease, Immune System and Environment. Allergy and Asthma Proceedings, 28, 410-417. http://dx.doi.org/10.1111/j.1365-2249.2009.04073.x

[16] Zulian, F., Falcini, F., Zancan, L., et al. (2003) Acute Surgical Abdomen as Presenting Manifestation of Kawasaki Disease. Journal of Pediatrics, 142, 731-735. http://dx.doi.org/10.1067/mpd.2003.232

[17] Dillon, M.J., Eleftheriou, D. and Brogan, P.A. (2010) Medium-Size-Vessel Vasculitis. Pediatric Nephrology, 25, 16411652. http://dx.doi.org/10.1007/s00467-009-1336-1

[18] Coustasse, A., Larry, J. and Lee, D. (2012) Can Kawasaki Disease Be Managed? Permanente Journal, 16, 70-72.

[19] Gomard-Mennesson, E., landron, C., Dauphin, C., et al. (2010) Kawasaki Disease in Adults: Report of 10 Cases. Medicine, 89, 149-158. http://dx.doi.org/10.1097/MD.0b013e3181df193c

[20] Burns, J.C. (2001) Kawasaki Disease. Advances in Pediatrics, 48, 157-177.

[21] Gedalia, A. (2002) Kawasaki Disease: An Update. Current Rheumatology Reports, 4, 25-29. http://dx.doi.org/10.1007/s11926-002-0020-2 